\title{
A numerical model to investigate the polarisation azimuth of ULF waves through an ionosphere with oblique magnetic fields
}

\author{
M. D. Sciffer, C. L. Waters, and F. W. Menk \\ School of Mathematical and Physical Sciences and CRC for Satellite Systems, The University of Newcastle, New South \\ Wales, Australia
}

Received: 27 May 2005 - Revised: 12 October 2005 - Accepted: 17 October 2005 - Published: 21 December 2005

\begin{abstract}
A one dimensional, computational model for the propagation of ultra low frequency (ULF; $1-100 \mathrm{mHz}$ ) wave fields from the Earth's magnetosphere through the ionosphere, atmosphere and into the ground is presented. The model is formulated to include solutions for high latitudes where the Earth's magnetic field, $\left(\boldsymbol{B}_{\mathbf{0}}\right)$, is near vertical and for oblique magnetic fields applicable at lower latitudes. The model is used to investigate the wave polarisation azimuth in the magnetosphere compared with the ground wave fields, as a function of the dip angle of $\boldsymbol{B}_{\mathbf{0}}$. We find that for typical ULF wave scale sizes, a $90^{\circ}$ rotation of the wave polarisation azimuth from the magnetosphere to the ground occurs at high latitudes. However, this effect does not necessarily occur at lower latitudes in all cases. We show that the degree to which the wave polarisation azimuth rotates critically depends on the properties of the compressional ULF wave mode.
\end{abstract}

Keywords. Ionosphere (Ionosphere-atmosphere interactions; Wave propagation) - Magnetospheric physics (Magnetosphere-ionosphere interactions)

\section{Introduction}

Ultra-low frequency (ULF) plasma waves in the $1-100 \mathrm{mHz}$ range are ubiquitous in the Earth's magnetosphere. Ground based sensors, such as sensitive magnetometers, detect these waves and the observations are used to deduce ULF wave generation and propagation mechanisms which in turn reveal the dynamics of the magnetosphere. For detection at the ground, ULF wave energy must pass through the ionosphere and atmosphere. A thorough understanding of ULF wave propagation through the ionosphere/atmosphere system is important for using ULF wave properties to remote sense the magnetosphere. Even though the wavelengths are much larger than the height of the ionosphere, there are

Correspondence to: C. L. Waters

(colin.waters@newcastle.edu.au) major alterations to the wave characteristics as they leave the highly ionoised medium in the magnetosphere and propagate through the neutral atmosphere to the ground.

The properties of ULF waves in the ionosphere and atmosphere have been examined for both vertical (or nearvertical) and horizontal orientations of $\boldsymbol{B}_{\mathbf{0}}$ (Hughes, 1974; Hughes and Southwood, 1976; Zhang and Cole, 1994). A combined analytic and numerical study of ULF wave propagation from the magnetosphere through the ionosphere to the ground was given by Hughes (1974) who examined the problem using the Maxwell equations. The analytic solutions used height integrated conductivities and approximated the ionosphere as a thin current sheet. Ideal MHD conditions were used to describe the behaviour of the waves above the ionosphere current sheet. Hughes (1974) examined the ULF wave fields for vertical and oblique geomagnetic fields appropriate to high latitude regions, and showed how the wave fields may be "screened" by the ionosphere. In particular, for an incident shear Alfvén mode ULF wave with the perturbation magnetic field in the east-west (y) direction in the magnetosphere, (i.e. $k_{x}=0$ ), the wave magnetic field at the ground was shown to be in the north-south (x) direction. One conclusion of Hughes (1974) was that wave reflection at the top of the ionosphere was very efficient with a reflection coefficient $\approx 1$. More recently, the ionosphere has been shown to behave more inductively for various frequencies, conductivity profiles and wavenumbers (Yoshikawa et al., 2002; Sciffer et al., 2004) which lead to smaller reflection coefficients.

Hughes (1974) also developed a numerical model which examined the case where the geomagnetic field was oblique and $k_{x} \neq 0$. However, the condition, $\frac{\omega}{V_{a}} \ll k_{\perp}$ was imposed and the polarisation of the shear Alfvén wave had no dependence on the vertical wave number, $k_{z}$, in the magnetosphere/ionosphere boundary condition. This makes the solutions valid only for near vertical $\boldsymbol{B}_{\mathbf{0}}$. For dip angles of the geomagnetic field corresponding to lower latitudes, there is a contribution of $k_{x}$ along the direction of $\boldsymbol{B}_{\mathbf{0}}$ which may be greater than $\frac{\omega}{V_{a}}$. Furthermore, in Hughes (1974) the 
wavenumber, $k$, was assumed to be $<\frac{1}{100} \mathrm{~km}^{-1}$ and the incident wave from the magnetosphere was limited to the shear Alfvén mode. In the present paper we investigate the ULF wave solutions with detailed altitude information for mid to low latitude geomagnetic field dip angles and allow for an inductive ionosphere, small wave numbers and incident compressional mode energy.

Zhang and Cole $(1994,1995)$ presented a numerical model of ULF wave propagation through the ionosphere for vertical and horizontal $\boldsymbol{B}_{\mathbf{0}}$ cases. They formulated the solution as a one dimensional (1-D), electromagnetic wave propagation model which was solved as a boundary value problem. Rather than impose ideal MHD conditions, the top boundary was assumed to be a uniform cold plasma with finite conductivity. This formulation allowed the incident wave mode mix to be specified in terms of the wave polarisation at the top boundary, with separation into upward and downward wave solutions calculated from constraints at the top boundary. Zhang and Cole (1995) examined the case where $\boldsymbol{B}_{\mathbf{0}}$ was horizontal, relating to equatorial regions where the formulation considered only the incident compressional mode wave. Zhang and Cole (1994) examined the case for a vertical geomagnetic field while allowing for a mixture of incident ULF wave modes. The magnetosphere medium was described by resistive plasma equations with finite conductivity in the topside region giving complex wave numbers and elliptically polarised waves. The effect of numerical swamping (e.g. Pitteway, 1965) due to the compressional mode was avoided by Hughes (1974) by limiting $k_{\perp} \leq \frac{1}{100} \mathrm{~km}^{-1}$. This ensured the evancesent decay length was large so that the growing compressional mode solution (which is unphysical) did not dominate the numerical solution. This condition was subsequently removed in Hughes and Southwood (1976) where the two wave modes were kept orthogonal.

The extension of modeling ULF wave propagation through the ionosphere for any dip angle of $\boldsymbol{B}_{\mathbf{0}}$ was presented as an analytic formulation by Sciffer and Waters (2002). The aim of the present paper is to extended that work for oblique $\boldsymbol{B}_{\mathbf{0}}$ to include a realistic height dependent conductivity tensor. We keep the finite conductivity plasma condition in the magnetosphere top boundary as described by Zhang and Cole (1994) and include non vertical $\boldsymbol{B}_{\mathbf{0}}$. The effect of oblique $\boldsymbol{B}_{\mathbf{0}}$ on the well known $90^{\circ}$ rotation in polarisation azimuth of the ULF wave field between the magnetosphere and ground is examined to determine if these effects remain valid for an oblique geomagnetic field geometry.

\section{Mathematical formulation}

A ULF wave incident from the magnetosphere may be described as an electromagnetic disturbance. We assume that the magnetosphere and ionosphere plasma is overall electrically neutral and that the zero order electric field, $\boldsymbol{E}_{\mathbf{0}}$, is zero. The relevant Maxwell equations are, $\nabla \times \boldsymbol{E}=-\frac{\partial \boldsymbol{B}}{\partial t}$

$\boldsymbol{\nabla} \times \boldsymbol{H}=\boldsymbol{J}+\frac{\partial \boldsymbol{D}}{\partial t}$

where the current density, $\boldsymbol{J}$ and magnetic flux density, $\boldsymbol{B}$ are given by

$\boldsymbol{J}=\bar{\sigma} \boldsymbol{E}$

$\boldsymbol{B}=\mu \boldsymbol{H}$

where $\bar{\sigma}$ is the conductivity tensor.

A Cartesian coordinate system is used where $X$ is northward, $Y$ is westward and $Z$ is radially outward from the surface of the Earth. The geomagnetic field, $\boldsymbol{B}_{\mathbf{0}}$, lies in the $X Z$ plane at an angle, $I$ to the horizontal. The ULF wave electric and magnetic perturbation fields are $\boldsymbol{e}$ and $\boldsymbol{b}$ respectively so that

$\boldsymbol{B}=\boldsymbol{B}_{0}+\boldsymbol{b}=\left(B_{0} \cos (I), 0, B_{0} \sin (I)\right)+\left(b_{x}, b_{y}, b_{z}\right)$

$\boldsymbol{E}=\boldsymbol{E}_{0}+\boldsymbol{e}=\left(e_{x}, e_{y}, e_{z}\right)$

Fourier analysing in time and space with an $e^{i\left(k_{x} x+k_{y} y-\omega t\right)}$ dependence, the governing equations in component form become

$$
\begin{aligned}
0= & \frac{i k_{y} \epsilon_{13}}{\epsilon_{33}} b_{x}-\left(\frac{\partial}{\partial z}+\frac{i k_{x} \epsilon_{13}}{\epsilon_{33}}\right) b_{y}-i\left[\frac{k_{y}^{2}}{\omega}-\frac{\omega}{c^{2}}\left(\epsilon_{11}-\frac{\epsilon_{31} \epsilon_{13}}{\epsilon_{33}}\right)\right] e_{x} \\
& +i\left[\frac{k_{x} k_{y}}{\omega}+\frac{\omega}{c^{2}}\left(\epsilon_{12}-\frac{\epsilon_{32} \epsilon_{13}}{\epsilon_{33}}\right)\right] e_{y}
\end{aligned}
$$

$$
\begin{aligned}
0= & \left(\frac{\partial}{\partial z}+\frac{i k_{y} \epsilon_{23}}{\epsilon_{33}}\right) b_{x}-\frac{i k_{x} \epsilon_{23}}{\epsilon_{33}} b_{y}+i\left[\frac{k_{x} k_{y}}{\omega}+\frac{\omega}{c^{2}}\left(\epsilon_{21}-\frac{\epsilon_{31} \epsilon_{23}}{\epsilon_{33}}\right)\right] e_{x} \\
& -i\left[\frac{k_{y}^{2}}{\omega}-\frac{\omega}{c^{2}}\left(\epsilon_{22}-\frac{\epsilon_{32} \epsilon_{23}}{\epsilon_{33}}\right)\right] e_{y}
\end{aligned}
$$

$$
\begin{aligned}
0= & \left(i \omega-\frac{c^{2} k_{y}^{2}}{\omega \epsilon_{33}}\right) b_{x}+\left(\frac{i c^{2} k_{x} k_{y}}{\omega \epsilon_{33}}\right) b_{y}+\frac{i k_{y} \epsilon_{31}}{\epsilon_{33}} e_{x} \\
& -\left(\frac{\partial}{\partial z}+\frac{i k_{y} \epsilon_{32}}{\epsilon_{33}}\right) e_{y}
\end{aligned}
$$

$$
\begin{aligned}
0= & \frac{i c^{2} k_{x} k_{y}}{\omega \epsilon_{33}} b_{x}+\left(i \omega-\frac{c^{2} k_{x}^{2}}{\omega \epsilon_{33}}\right) b_{y}-\left(\frac{\partial}{\partial z}+\frac{i k_{x} \epsilon_{31}}{\epsilon_{33}}\right) e_{x} \\
& +\left(\frac{i k_{x} \epsilon_{32}}{\epsilon_{33}}\right) e_{y} .
\end{aligned}
$$

This is a system of four, first order differential equations involving the spatial derivatives in altitude, $z$. The $e_{z}$ and $b_{z}$ wave components are given by

$$
\begin{aligned}
& e_{z}=\frac{-k_{y} c^{2}}{\omega \epsilon_{33}} b_{x}+\frac{k_{x} c^{2}}{\omega \epsilon_{33}} b_{y}-\frac{\epsilon_{31}}{\epsilon_{33}} e_{x}-\frac{\epsilon_{32}}{\epsilon_{33}} e_{y} \\
& b_{z}=\frac{k_{y}}{\omega} e_{x}-\frac{k_{x}}{\omega} e_{y}
\end{aligned}
$$


The $\epsilon_{i j}$ are the $i$ th row and $j$ th column of the dielectric tensor, $\bar{\epsilon}$, which is related to the conductivity tensor by

$\bar{\epsilon}=\bar{I}-\frac{i}{\epsilon_{0} \omega} \bar{\sigma}$

where $\bar{I}$ is the identity tensor.

In specifying the boundary conditions we assume that the plasma above the region where the equations are solved is cold and uniform but has finite conductivity. An examination of the wave modes which can propagate above the ionosphere and traverse the upper boundary is in order. The nature of the wave modes which can exist in a magnetised cold plasma has been examined by a number of authors (e.g. Stix, 1962; Pitteway, 1965; Zhang and Cole, 1994). Since our model assumes a resistive plasma, there are no pure shear Alfvén (transverse) or pure fast (compressional) wave modes in the MHD sense. However, we will identify the wave mode that has the majority of its Poynting flux in the direction of $\boldsymbol{B}_{\mathbf{0}}$ as the "shear Alfvén" mode and the wave with the majority of its Poynting flux in the direction of $\boldsymbol{k}_{f}$ as the "compressional" or fast mode.

Taking the curl of (1) and using (2), the matrix which defines the dispersion relation for the resistive plasma is

$$
\left(\boldsymbol{k} \cdot \boldsymbol{k}-k^{2} \bar{I}+k_{0}^{2} \bar{\epsilon}\right) \cdot \boldsymbol{E}=0
$$

Alternatively, Eq. (14) may be written in terms of a matrix, $\Psi\left(k_{z}\right)$ as

$\Psi\left(k_{z}\right) \cdot \boldsymbol{E}=0$

where $\boldsymbol{k}=i\left(k_{x}, k_{y}, k_{z}\right)$ and $k_{0}=\omega\left(\mu_{0} \epsilon_{0}\right)^{\frac{1}{2}}$, the free space wavenumber.

When $k_{x}$ and $k_{y}$ are specified, the determinant of $\Psi$ may be written as a quartic in $k_{z}$. For non-trivial solutions the determinant of $\Psi$ must be zero. The four roots of $\operatorname{det}(\Psi)=0$ represent the vertical wavenumbers for each wave mode which may exist in the region above the computational domain. Denoting the direction set (incident or reflected) by $d$ and the wave mode set (Alfve'n or fast) by $m$ then for each root, $k_{z, m}^{d}$, we may solve for the electric field, expressed in unit vector form as described in the next section. The characteristic equation is

$\Psi\left(k_{z, m}^{d}\right) \cdot \boldsymbol{P}=\lambda \boldsymbol{P}=0$

where $\lambda$ is the eigenvalue of the matrix $\Psi$ evaluated at each of the roots, $k_{z, m}^{d}$. The unit magnitude eigenvector $\hat{\boldsymbol{P}}_{m}^{d}$ associated with $\lambda=0$ represents the polarisation of the electric field of the corresponding wave mode, $m$. We shall denote shear Alfv́en wave modes as $\hat{\boldsymbol{P}}_{a}^{r}$ and $\hat{\boldsymbol{P}}_{a}^{i}$, where the $i$ and $r$ indicate whether the vertical Poynting flux is towards or away from the surface of the earth. Similarly, an incident and reflected compressional wave mode are identified as $\hat{\boldsymbol{P}}_{f}^{i}$ and $\hat{\boldsymbol{P}}_{f}^{r}$, respectively.
The compressional wave mode solutions for $\operatorname{det}(\Psi)=0$ for a given $k_{x}, k_{y}$ and conductivity profile, are denoted as $k_{z, f}^{r}$ and $k_{z, f}^{i}$ and are generally complex. The real part indicates the propagating nature of the mode while the imaginary part indicates the spatial scale of the wave mode. For ideal MHD conditions, the compressional mode dispersion relation gives vertical wavenumbers which are purely imaginary, an "evanescent" mode or purely real, a "propagating" mode. For complex $k_{z}$, the waveform oscillates while diminishing with distance. In the following sections the wave frequencies are $<100 \mathrm{mHz}$ and we find that the solution of $\operatorname{det}(\Psi)=0$ for the compressional mode vertical wavenumber is similar to the ideal MHD case. Therefore, we use the ideal MHD terminology where the fast mode is 'propagating' if $\left|\operatorname{Re}\left(k_{z, f}\right)\right| \gg\left|\operatorname{Im}\left(k_{z, f}\right)\right|$ and "evanescent" if $\left|\operatorname{Re}\left(k_{z, f}\right)\right| \ll\left|\operatorname{Im}\left(k_{z, f}\right)\right|$. Also, similiar to ideal MHD, for a fixed $k_{x}, k_{y}$ and conductivity there is a "critical frequency" at which the vertical wavenumber of the fast mode transitions between "propagating" and "evanescent".

The top boundary includes any mixture of shear Alfvén and compressional mode disturbances. The total electric field $\boldsymbol{E}_{\boldsymbol{t}}$, is comprised of both downward (incident) and upward (reflected) components of both wave modes so that the total electric field at the top of the computational domain is given by

$$
\left(\begin{array}{l}
e_{x} \\
e_{y} \\
e_{z}
\end{array}\right)=\alpha^{r} \hat{\boldsymbol{P}}_{a}^{r}+\alpha^{i} \hat{\boldsymbol{P}}_{a}^{i}+\beta^{r} \hat{\boldsymbol{P}}_{f}^{r}+\beta^{i} \hat{\boldsymbol{P}}_{f}^{i}
$$

The $\alpha$ and $\beta$ are amplitude factors for each of the wave modes entering or leaving the solution region. This superposition of the magnetosphere electric fields allows the wave fields and their derivatives to be expressed in terms of the composition of MHD wave modes present above the top boundary. The polarization of each wave mode is contained in the $\hat{\boldsymbol{P}}$.

Given that we have linearized the problem in the form $e^{i\left(k_{x} x+k_{y} y\right)}$, we may write the $\mathrm{x}$ and $\mathrm{y}$ components of the total electric field and the derivatives as

$$
\begin{aligned}
& E_{x}=\alpha^{i} \hat{P_{x, a}^{i}}+\beta^{i} \hat{P_{x, f}^{i}}+\alpha^{r} P_{x, a}^{r}+\beta^{r} P_{x, f}^{\wedge} \\
& E_{y}=\alpha^{i} \hat{P_{y, a}^{i}}+\beta^{i} \hat{P_{y, f}^{i}}+\alpha^{r} \hat{P_{y, a}^{r}}+\beta^{r} \hat{P_{y, f}^{r}}
\end{aligned}
$$

$$
\begin{gathered}
\frac{\partial E_{x}}{\partial z}=i k_{z, a}^{i} \alpha^{i} \hat{P_{x, a}^{i}}+i k_{z, f}^{i} \beta^{i} \hat{P}_{x, f}^{\hat{i}} \\
+i k_{z, a}^{r} \alpha^{r} \hat{P_{x, a}^{r}}+i k_{z, f}^{r} \beta^{r} \hat{P}_{x, f}^{\hat{r}}
\end{gathered}
$$

$$
\begin{gathered}
\frac{\partial E_{y}}{\partial z}=i k_{z, a}^{i} \alpha^{i} \hat{P_{y, a}^{i}}+i k_{z, f}^{i} \beta^{i} P_{y, f}^{\hat{i}} \\
+i k_{z, a}^{r} \alpha^{r} \hat{P}_{y, a}^{r}+i k_{z, f}^{r} \beta^{r} P_{y, f}^{\wedge}
\end{gathered}
$$


The total electric field and its derivatives can now be written in terms of the matrix $\Omega$, where

$$
\begin{aligned}
& {\left[\begin{array}{c}
E_{x} \\
E_{y} \\
\frac{\partial E_{x}}{\partial z} \\
\frac{\partial E_{y}}{\partial z}
\end{array}\right]=[\Omega] \cdot\left[\begin{array}{c}
\alpha^{i} \\
\beta^{i} \\
\alpha^{r} \\
\beta^{r}
\end{array}\right]=}
\end{aligned}
$$

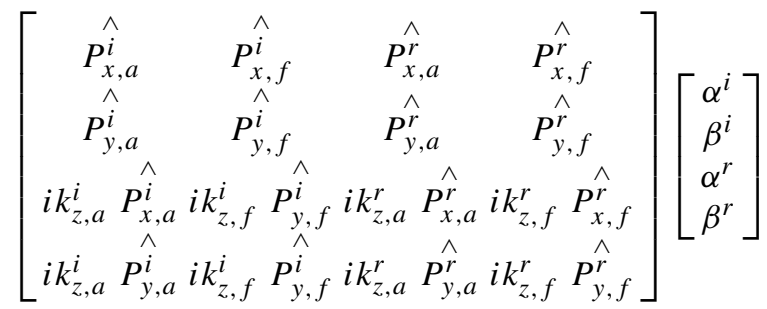

To obtain the upward and downward components of the total wave electric field we need to invert the matrix $\Omega$ so that

$$
\left[\begin{array}{c}
\alpha^{i} \\
\beta^{i} \\
\alpha^{r} \\
\beta^{r}
\end{array}\right]=[\Omega]^{-1} \cdot\left[\begin{array}{c}
E_{x} \\
E_{y} \\
\frac{\partial E_{x}}{\partial z} \\
\frac{\partial E_{y}}{\partial z}
\end{array}\right]
$$

The incident wave amplitude $\left(\alpha^{i}\right.$ and $\left.\beta^{i}\right)$ can now be expressed in terms of the total electric field and used to specify the upper boundary conditions:

$$
\begin{aligned}
\alpha^{i} & =\Omega^{d a}\left(E_{x}, E_{y}, \frac{\partial E_{x}}{\partial z}, \frac{\partial E_{y}}{\partial z}\right) \\
\beta_{i} & =\Omega^{d f}\left(E_{x}, E_{y}, \frac{\partial E_{x}}{\partial z}, \frac{\partial E_{y}}{\partial z}\right)
\end{aligned}
$$

where $\Omega^{d a}$ and $\Omega^{d f}$ are the corresponding rows of the matrix $\Omega^{-1}$. This requires values for the incident amplitudes of both wave modes. In specifying $\alpha^{i}$ and $\beta^{i}$ we also specify the mode mix of the incident disturbance. An advantage of this procedure is that the expression for the amplitude of the reflected modes at the top boundary, $\alpha^{r}$ and $\beta^{r}$ may be calculated from Eq. (23) after the solution for $\boldsymbol{E}_{\boldsymbol{t}}$ has been obtained.

Since (7) to (10) describe a system of four equations, a further two boundary conditions are required. For these we assume the Earth is a uniform, homogeneous conductor with finite conductivity which implies

$$
\begin{aligned}
& \frac{\partial e_{x}}{\partial z}-\gamma\left(\sigma_{g}, k_{x}, k_{y}, \omega\right) e_{x}=0 \\
& \frac{\partial e_{y}}{\partial z}-\gamma\left(\sigma_{g}, k_{x}, k_{y}, \omega\right) e_{y}=0
\end{aligned}
$$

where $\gamma$ sets the ground to be a uniform, homogeneous conductor with conductivity $\sigma_{g}$. The solutions which satisfy this condition are imposed so that the ULF wave is attenuated beneath the surface of the Earth. For the results presented in this paper we have set $\sigma_{g}=10^{-2} \mathrm{Mho} / \mathrm{m}$.

With these boundary conditions, and a second order finite differencing scheme we solved the boundary value problem using the Numerical Algorithms Group (NAG) package number FO4ADF. The computational domain was chosen
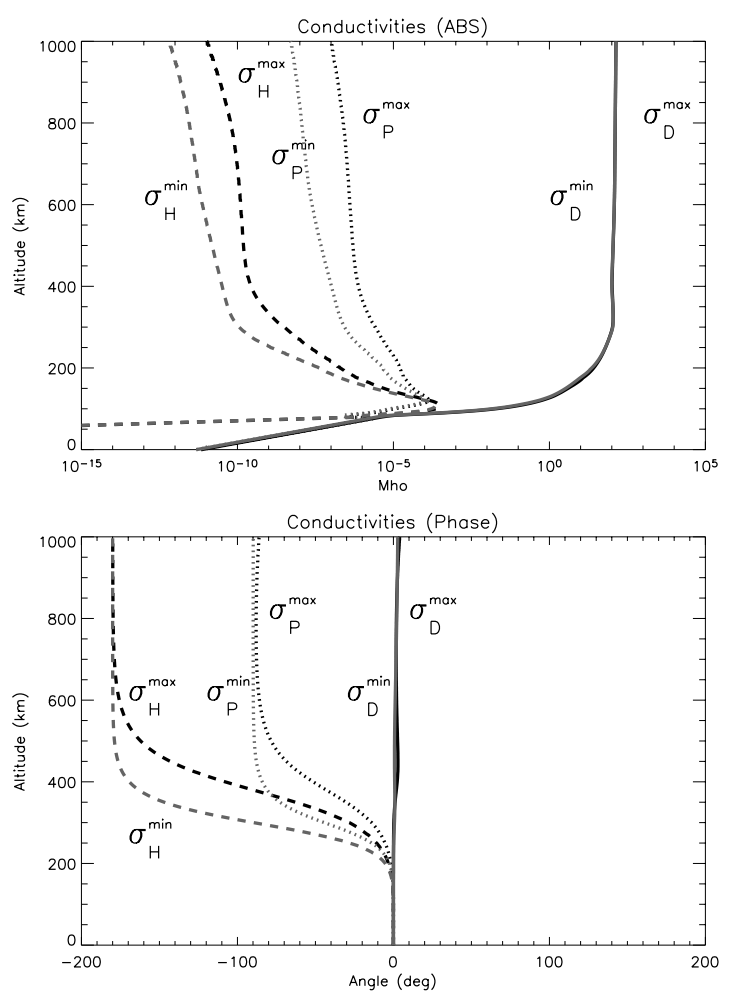

Fig. 1. The direct (solid), Pedersen (dotted)) and Hall (dashed) conductivities for solar minimum and maximum following Hughes (1974). An incident $16 \mathrm{mHz}(\omega=0.1) \mathrm{ULF}$ wave was used.

to be $1000 \mathrm{~km}$ in altitude with a uniform grid spacing of $2 \mathrm{~km}$. The composition of the atmosphere and ionosphere were calculated from the thermosphere model based on satellite mass spectrometer and ground-based incoherent scatter data (MSISE90), (Hedin, 1991) and the International Reference Ionosphere (IRI-95) models. The International Geomagnetic Reference Field (IGRF-95) was used to provide values for $\boldsymbol{B}_{\mathbf{0}}$. Data for the collision frequencies below $80 \mathrm{~km}$ were extrapolated to the ground using the expressions in Appendix A. The eigenvectors, $\mathbf{P}$, in Eq. (16) were found using the NAG routine F02GBF, while the roots of Eq. (14) were computed using the NAG routine C02AFF.

\section{ULF wave polarisation azimuth in the horizontal plane}

Validation of the computations was achieved by comparing with published results and with the analytic solutions developed by Sciffer and Waters (2002) and Sciffer et al. (2004). In this section, results from a comparison with those of Hughes (1974) are presented, followed by a study of the horizontal polarisation of the magnetic perturbations of ULF waves through the ionosphere as a function of latitude.

The first step for the computations is to define the wave propagation medium using the IRI-95 and MSISE90 ionosphere and thermosphere models. For comparison with Hughes (1974), we simulate midday, sunspot minimum and 
maximum conditions, ULF wave frequency of $\omega=0.1$ and a $\boldsymbol{B}_{0}$ dip angle, $\mathrm{I}=70^{\circ}$. For these conditions, the direct, Pedersen and Hall conductivities, including the kinetic terms are shown in Fig. 1. Above $200 \mathrm{~km}$, the conductivity along $\mathbf{B}_{0}$ (direct conductivity) is of order $1 \times 10^{7}$ times larger than the Pedersen and Hall conductivities. However, the Pedersen and Hall terms give rise to the anisotropic ionosphere conductivity, allowing conversion of the ULF modes. The conductivity has been "ramped" down so that it decreases with decreasing altitude in the atmosphere as described by Hughes (1974). The small but finite conductivity in the atmosphere is discussed further in Sect. 4.

Figure 2 shows the electric and magnetic ULF wave fields for an incident shear Alfvén-like wave mode. While the details of the ionosphere used in Hughes (1974) are not provided, the wave fields are very similar to his Fig. 4. Computation of our model using the same parameters as other examples presented in Hughes (1974) also agreed with their results. Results from our model were also compared with those in Zhang and Cole (1994), Hughes and Southwood (1976) and Poole et al. (1988) and found to reproduce the respective published plots. This shows that for the condition that specifies an incident shear Alfvén mode only, there is little difference between assuming that the medium at the top boundary consists of a resistive plasma compared with ideal MHD conditions. This also implies that at these frequencies, the ideal MHD formulation in the analytic model of Sciffer and Waters (2002) is a reasonable approximation. For reference, the electric fields of the ULF waves from Sciffer and Waters (2002) are

$e^{f}=\beta\left[-k_{y} \sin (I), k_{x} \sin (I)-k_{z, f} \cos (I), k_{y} \cos (I)\right]$

for the compressional mode and

$$
\begin{aligned}
e^{a}=\alpha & \alpha\left(k_{x} \sin (I)-k_{z, a} \cos (I)\right) \sin (I), \\
& \left.k_{y},-\left(k_{x} \sin (I)-k_{z, a} \cos (I)\right) \cos (I)\right]
\end{aligned}
$$

for the shear Alfvén mode. The 90-deg rotation (NDR) effect of wave polarisation azimuth described by Hughes (1974) can be seen in the azimuth angle shown in Fig. 2.

\section{Polarisation azimuth for a vertical $\boldsymbol{B}_{\mathbf{0}}$}

Figure 2 shows that the ULF magnetic wave fields change in amplitude and phase as a function of altitude. A well known property of ULF wave fields is the polarisation azimuth in the horizontal plane, calculated using the wave polarisation parameters from $b_{x}$ and $b_{y}$. The simplest case is for vertical $\boldsymbol{B}_{\mathbf{0}}$ and small $k_{x}$. Figure 3a shows the classic case where an incident shear Alfvén wave with $k_{x}=1 \times 10^{-10} \mathrm{~m}^{-1}$ and $k_{y}=1 \times 10^{-6} \mathrm{~m}^{-1}$ is modeled. The Alfvén speed at $1000 \mathrm{~km}$ altitude is $2.8 \times 10^{6} \mathrm{~ms}^{-1}$. This gives $k_{z, a}=\omega / V_{a}=3.6 \times 10^{-8} \mathrm{~m}^{-1}$ for the incident shear Alfvén mode. From Eqn. (29), the incident shear Alfvén mode has an electric field vector proportional to $e^{a}=\left(k_{x}, k_{y}, 0\right)$ with the larger $e_{y}$ clearly seen in Fig. 3a. Therefore, the magnetic perturbation associated with the shear Alfvén mode is largely in the $b_{x}$ component and the compressional mode has $b_{y}$ and $b_{z}$ components.
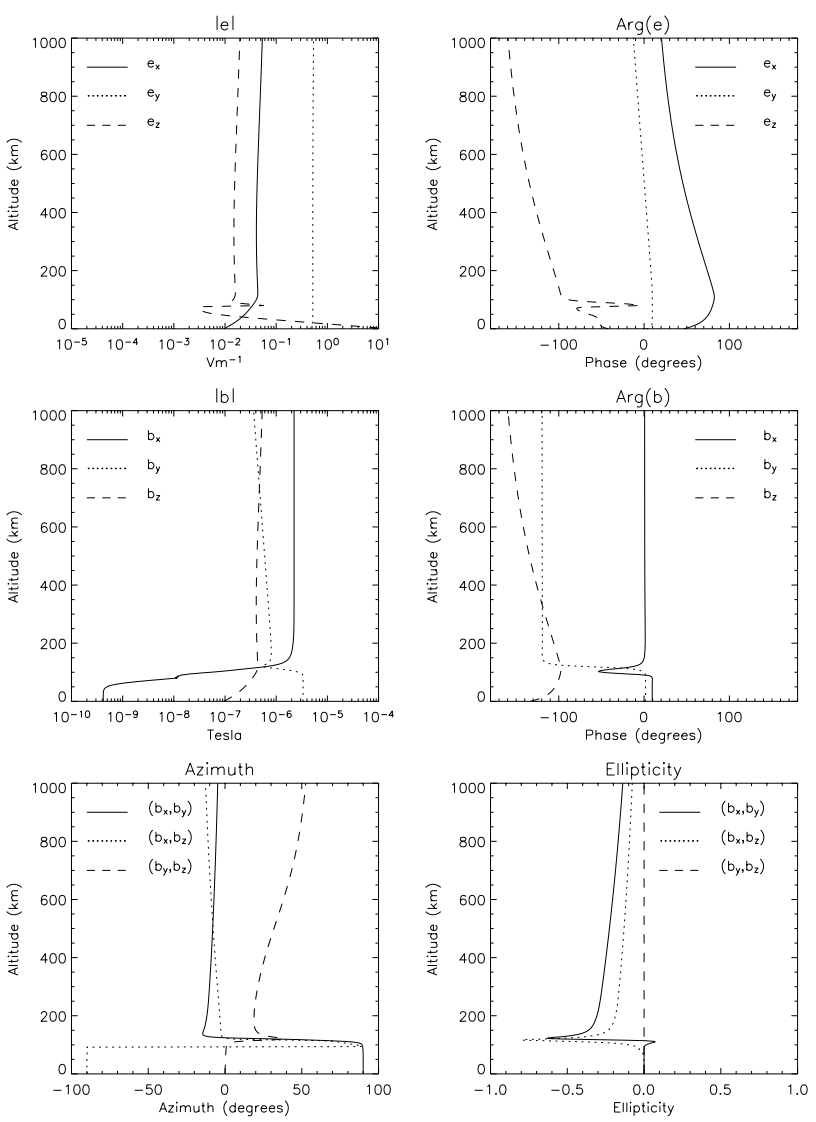

Fig. 2. ULF wave fields and polarisation azimuth for $k_{x}=10^{-10} \mathrm{~m}^{-1}, k_{y}=10^{-6} \mathrm{~m}^{-1}$ and $\omega=0.1(f \approx 16 \mathrm{mHz})$. The dip angle is $70^{\circ}$. Compare with Fig. 4. in Hughes (1974).

Comparing with Fig. 2, the $e_{x}$ and $e_{y}$ components of the electric field in Fig. 3a are similiar, while the vertical electric field differs due to the inclination of the background magnetic field in Fig. 2. For vertical $\boldsymbol{B}_{\mathbf{0}}, e_{z} \approx 0$ since the field aligned component of the electric field is small where the direct conductivity is large. The ULF wave magnetic fields vary in a similar fashion compared with those in the oblique field in Fig. 2. This is due to $e_{z} \approx 0$ for the vertical field and some small differences in the phasing of the fields. The polarisation azimuth rotation in the $b_{x}$ and $b_{y}$ components in both Figs. 2 and $3 a$ are very similiar. The explanation for this effect in terms of $\nabla \times \boldsymbol{b}$ has been given by Southwood and Hughes (1983). This polarisation rotation of the wave fields occurs over a narrow range in altitude near $120 \mathrm{~km}$. The wave goes from linearly polarized to elliptically polarised in a right handed (clockwise) sense near $120 \mathrm{~km}$ altitude returning to linear polarisation in the atmosphere.

Figure $3 \mathrm{~b}$ has an incident shear Alfven wave where $k_{x}=1 \times 10^{-10} \mathrm{~m}^{-1}$ and $k_{y}=1 \times 10^{-5} \mathrm{~m}^{-1}$. The shorter wavelength in the east-west direction has a number of significant effects on the electric and magnetic wave fields. Comparing Figs. $3 \mathrm{a}$ and $3 \mathrm{~b}$, the $e_{x}, b_{y}$ and $b_{z}$ components (compressional mode) are significantly attenuated at higher altitudes for the shorter wavelength. Interaction within the 

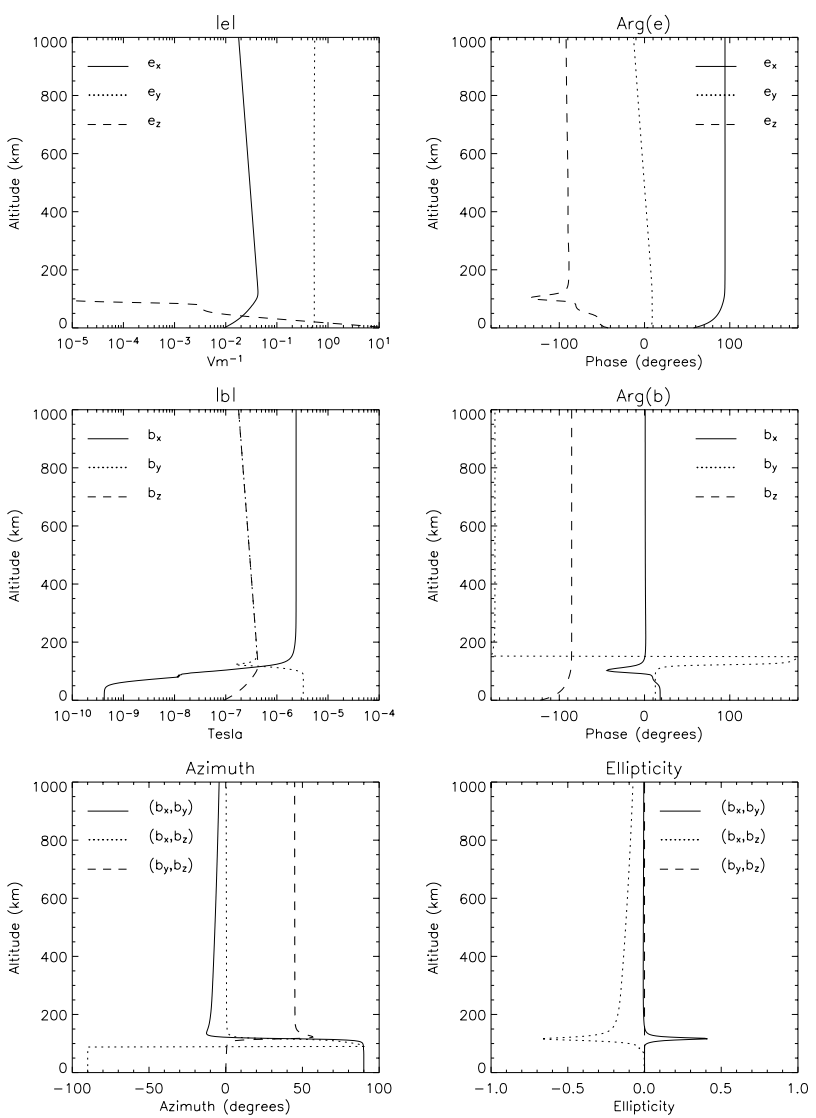

(a)
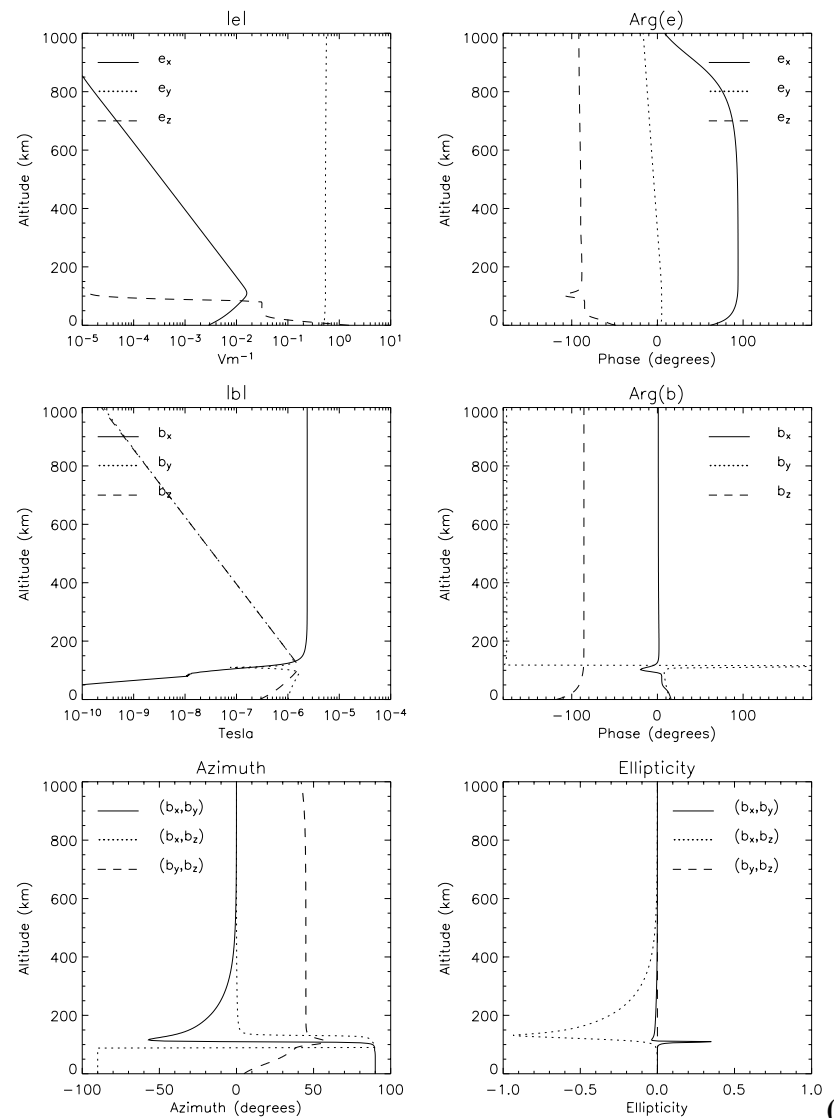

(b)

Fig. 3. Polarisation azimuth computed from $b_{x}$ and $b_{y}$ for a vertical $\boldsymbol{B}_{\mathbf{0}}$. In (a) $k_{x}=10^{-10} \mathrm{~m}^{-1}$ and $k_{y}=10^{-6} \mathrm{~m}^{-1}$, similar to Fig. 2 while in (b) $k_{x}=10^{-10} \mathrm{~m}^{-1}$ and $k_{y}=10^{-5} \mathrm{~m}^{-1}$ for frequency of $f=16 \mathrm{mHz}$ at solar minimum with $V_{a}=2.8 \times 10^{6} \mathrm{~ms}-1$ at $1000 \mathrm{~km}$ altitude. The wave fields are X (solid), Y (dotted) and Z (dashed).

ionosphere/atmosphere results in wave reflection and the resulting ionosphere current system gives rise to a compressional mode which forms a surface wave in this region (Yoshikawa and Itonaga, 2000; Sciffer and Waters, 2002). The evanescent nature of the compressional mode for the specified scale size is shown in the decay in amplitude with altitude. The wave field components of the shear Alfvén mode and the phasing of the fields are not significantly altered. The polarisation azimuth rotation in the horizontal magnetic field components also shows a NDR. However, in this case the NDR develops over an altitude range starting at $500 \mathrm{~km}$ and reaching $90^{\circ}$ near $120 \mathrm{~km}$, the ionospheric Eregion. Therefore, a straightforward rotation of ULF magnetic fields projected onto the ground, by rotating the magnetic field through $90^{\circ}$ may not be appropriate. This effect is particularly pertinent when using radar or low Earth orbit satellite data where measurements in space may be located within the region where the NDR has partially begun. The ellipticity is not significantly altered by the more gradual change in polarisation azimuth with altitude in Fig. 3b.

Figure 3 has shown that the ULF wavenumber affects the altitude range over which the NDR occurs. The polarisation azimuth computed from the $b_{x}$ and $b_{y}$ ULF wave fields as a function of both altitude and wavenumber in a vertical $\boldsymbol{B}_{0}$ is shown in Fig. 4a for solar maximum conditions where $V_{a}=6.5 \times 10^{5} \mathrm{~ms}^{-1}$ at $1000 \mathrm{~km}$ altitude. The incident shear Alfvén like wave has a dominant $b_{x}$ component. The major axis of the horizontal polarisation ellipse changes as a function of altitude and wavenumber above the ionosphere $\mathrm{E}$ region. Below this, the orientation of the major axis is rotated into the positive $y$ direction (west). As $k_{y}$ increases the vertical nature of the compressional mode changes from propagating to evanescent. From Eq. (14) the vertical wavenumber of the compressional mode at $1000 \mathrm{~km}$, in this case, is predominately propagating for $k_{y} \leq 1.5 \times 10^{-7} \mathrm{~m}^{-1}$.

In the regions where the compressional mode is propagating (small wavenumber) or highly evanescent (large wavenumber), the NDR transition occurs over a small altitude range. However, in the region where the wavenumbers vary between $2 \times 10^{-7} \mathrm{~m}^{-1}$ and $3 \times 10^{-5} \mathrm{~m}^{-1}$ the NDR develops over a larger range of altitudes. This corresponds with the case in Fig. 3b where the development of the NDR over a range of altitudes is due to the presence of the compressional wave mode component in $b_{y}$ being attenuated as a function of increasing altitude according to the $e$ folding distance of the evanescant compressional mode. 

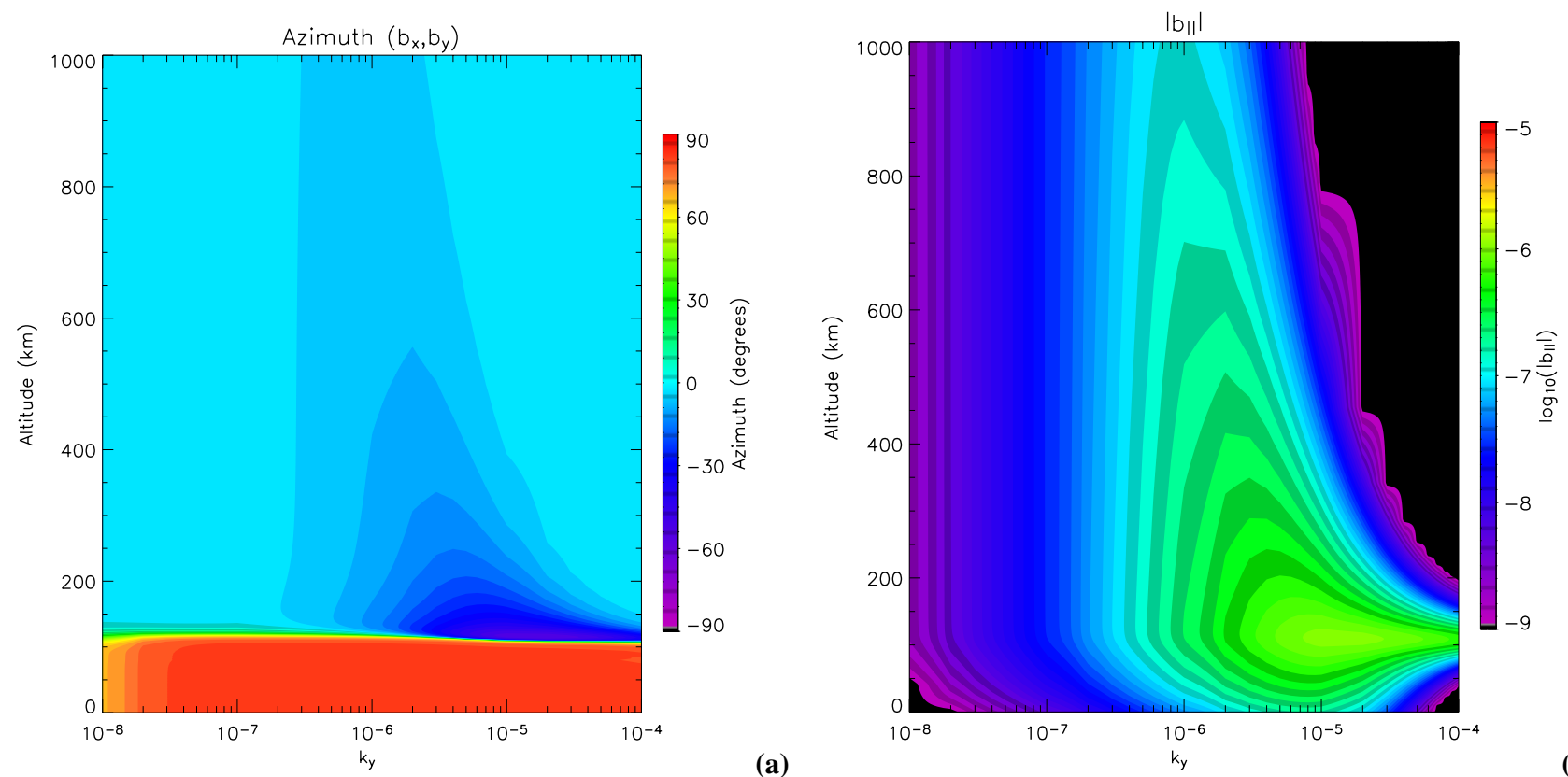

(b)

Fig. 4. (a) The polarisation azimuth computed from $b_{x}$ and $b_{y}$, for a frequency of $16 \mathrm{mHz}$ and dip angle of $I=90^{\circ}$ at solar maximum. The wavenumbers are $k_{x}=10^{-10} \mathrm{~m}^{-1}$ and $k_{y}$ varying between $10^{-8}$ and $10^{-4} \mathrm{~m}^{-1}$. (b) The amplitude of the field aligned (compressional) component of the ULF wave magnetic field $\left(b_{z}\right.$ in the case of a vertical field) for the parameters used in (a).

In order to see this effect, Fig. $4 \mathrm{~b}$ shows the field aligned component of the ULF wave magnetic field, which is $b_{z}$ for a vertical $\boldsymbol{B}_{\mathbf{0}}$. The $b_{z}$ component is a good indicator of how much compressional mode is present at a given altitude. The region where the compressional mode is generated can be seen to be near the ionosphere E-region where the Pedersen and Hall conductivities are large. As the wave number increases, the amplitude of the compressional magnetic field at $120 \mathrm{~km}$ altitude increases until it achieves a maximum value at $k_{y} \approx 1 \times 10^{-5} \mathrm{~m}^{-1}$. The amount of compressional mode as a function of altitude depends on the conversion coefficient, $|A F|$ (Sciffer and Waters, 2002) of the wave modes and the $e$ folding distance of the vertically evanescant compressional mode. Figure $4 \mathrm{~b}$ shows this behavior as a reduction in amplitude of $b_{z}$ above (and below) the ionosphere E-region for the larger values of the horizontal wavenumber. The important factors are the amount of converted compressional mode and the $e$ folding distance of the mode that determine the height at which the NDR will start, as well as the altitude dependence of the polarisation ellipse major axis rotation for the ULF wave. Since the rotation depends on the conversion between the ULF wave modes, parameters such as the ionosphere conductivities, wave frequencies and dip angle play an important part in determining these factors, as discussed in Sciffer et al. (2004).

\section{Polarisation azimuth for Oblique $B_{0}$}

The effect of a non vertical $\boldsymbol{B}_{\mathbf{0}}$ on the ULF wave polarisation azimuth for solar maximum ionosphere conditions is shown in Fig. 5a. The parameters are the same as those for Fig. 4a except that the inclination of $\boldsymbol{B}_{\mathbf{0}}$ is now $70^{\circ}$. The change in azimuth with altitude and wavenumber has features in common with Fig. 4a, particularly for wavenumbers greater than $k_{y} \approx 2 \times 10^{-7} \mathrm{~m}^{-1}$. The slight differences are due to the dependence of the wave mode conversion coefficients on the $\boldsymbol{B}_{\mathbf{0}}$ dip angle as explained by Sciffer and Waters (2002). However, for small wavenumbers, we have the possibility that the NDR does not occur. For the parameters chosen, this is for horizontal wavenumbers, $k_{y}<2 \times 10^{-7} \mathrm{~m}^{-1}$, where the compressional mode is propagating. The polarisation azimuth does not change with altitude above the ionosphere E-region, as the $b_{y}$ wave component does not decrease in amplitude with height.

Below $120 \mathrm{~km}$, we need to address the NDR effect as explained in Southwood and Hughes (1983). Basically, the reasoning considered $\nabla \times \boldsymbol{b}=0$ in the neutral atmosphere compared with $\nabla \times \boldsymbol{b} \neq 0$ in the magnetosphere. The change in $\nabla \times \boldsymbol{b}$ from the magnetosphere through to the atmosphere is manifested by a rotation of the wave fields by $90^{\circ}$. The curl of the horizontal magnetic wave fields is

$(\nabla \times \boldsymbol{b})_{z}=\mu_{0} j_{z}-i \frac{\omega}{c^{2}} e_{z}$

where $\boldsymbol{j}_{z}$ is the vertical conduction current density. However if the magnitude of $\boldsymbol{j}_{\boldsymbol{z}}$ in the atmosphere is comparable with $\left|(\nabla \times \boldsymbol{b})_{z}\right|$ in the magnetosphere, the polarisation azimuth will not exhibit the NDR. For small wavenumbers, $\boldsymbol{j}_{z}$ in the magnetosphere becomes comparable with the conduction current in the "neutral" atmosphere, due to the assumed decrease in conductivities with altitude in the atmosphere as 

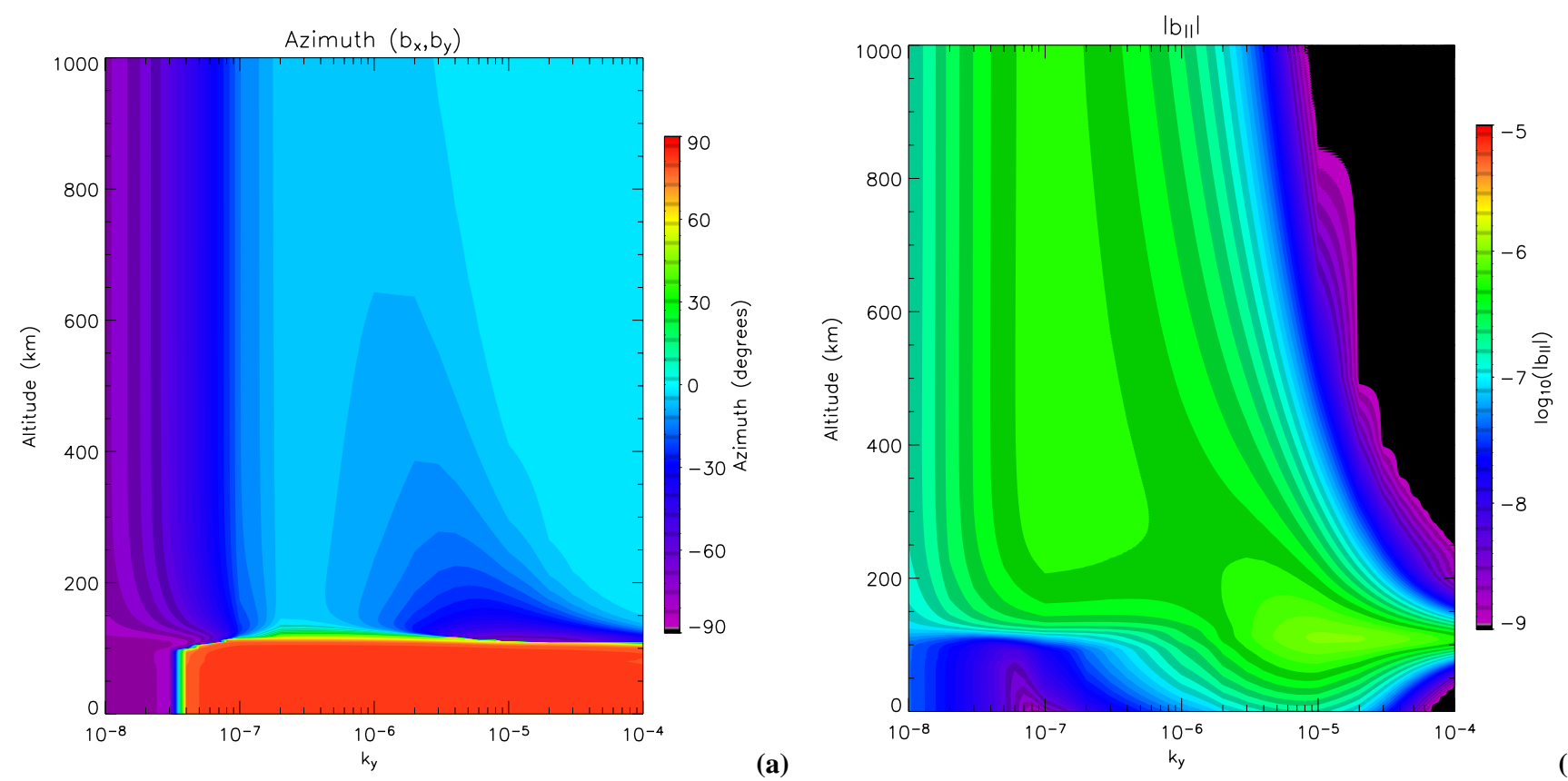

(b)

Fig. 5. Polarisation azimuth between the ULF wave $b_{x}$ and $b_{y}$ components for a frequency of $16 \mathrm{mHz}$, dip angle of $I=70^{\circ}$ and solar maximum ionosphere conditions. (a) Wavenumbers are $k_{x}=10^{-10} \mathrm{~m}^{-1}$ and $k_{y}$ varying between $10^{-8} \mathrm{~m}^{-1}$ and $10^{-4} \mathrm{~m}^{-1}$. (b) The amplitude of the field aligned (compressional) component of the ULF wave magnetic field for the parameters in (a).

shown in Fig. 1. To remedy this situation, the conductivities were ramped down more steeply than those in Hughes (1974) in the lower regions $(\leq 60 \mathrm{~km})$ of the atmosphere. This reduced the conduction current produced by the ULF wave in the atmosphere and the NDR was restored for the small wavenumbers in Fig. 5.

Figure $5 b$ shows the amplitude of the ULF wave magnetic field component that is aligned along $\boldsymbol{B}_{\mathbf{0}}$. Compared to the vertical case shown in Fig. 4b, more compressional mode appears for the smaller wavenumbers. Since the compressional mode at these wavenumbers is propagating, or has a large $e$ folding distance, there is a greater proportion of compressional mode energy from $120 \mathrm{~km}$ altitude and higher for non vertical dip angles. As the wavenumber increases the evanescent nature of the mode becomes important and the amplitude of the field aligned ULF wave magnetic field decreases exponentially, similar to the vertical $\boldsymbol{B}_{\mathbf{0}}$ case.

\section{Polarisation azimuth rotation for constant azimuthal wavenumber}

A common parameter used to describe the propagation properties of ULF waves is the azimuthal wavenumber, also known as the $m$ number. From Olson and Rostoker (1978),

$k_{y}=\frac{m}{2 \pi R_{E} \cos \left(\theta_{g}\right)}$

where $R_{e}$ is the Earth radius and $\theta_{g}$ is the geographic latitude. For example, for $m=3$ at mid latitudes $\left(\theta_{g} \approx 50^{\circ}\right)$, $k_{y} \approx 1 \times 10^{-7} \mathrm{~m}^{-1}$ while the $\boldsymbol{B}_{0}$ dip angle, $I \approx 80^{\circ}$. In order to gain some insight into the latitudinal dependence of the
ULF wave polarisation azimuth, the polarisation azimuth rotation was calculated as a function of altitude and geographic latitude for a constant wave frequency and constant $m$. The ionosphere conductivities were calculated at each latitude using the IRI-95 model for 10:00 LT on 28 September 2000 along the $150^{\circ}$ geographic longitude.

Figure 6 a shows the results for $f=25 \mathrm{mHz}$ and $m=3$. The ULF wave polarisation azimuth rotation depends on geographic latitude, over the $-70^{\circ}$ to $-20^{\circ}$ latitude range, $k_{y}$ varies from $2 \times 10^{-7}$ to $8 \times 10^{-8} \mathrm{~m}^{-1}$ at the lowest latitude. The conductivities were similar across all latitudes. The critical frequency at which the compressional mode starts to propagate for these latitudes is $\approx 20 \mathrm{mHz}$. The non-NDR effect at low latitudes depends on the conversion coefficients of the wave modes, a feature that is discussed later. If the ULF wave frequency is below the critical frequency, the ULF wave undergoes a NDR at all latitudes. Figure $6 \mathrm{~b}$ shows the results for $m=12$. The critical frequency is now shifted up to $\approx 80 \mathrm{mHz}$. This gives the usual NDR effect at all latitudes with a sharp change near the ionosphere E-region since the compressional wave mode is highly evanescent for these parameters.

Figure 6 illustrates an important consideration when projecting ULF wave magnetic (or electric) field measurements taken above the ionosphere and applying the NDR effect in mapping these fields down to the ground. Depending on ionospheric conditions, altitude and vertical wave structure the wave may not necessarily exhibit a NDR. While the results presented in Figs. 4-6 have been for solar 

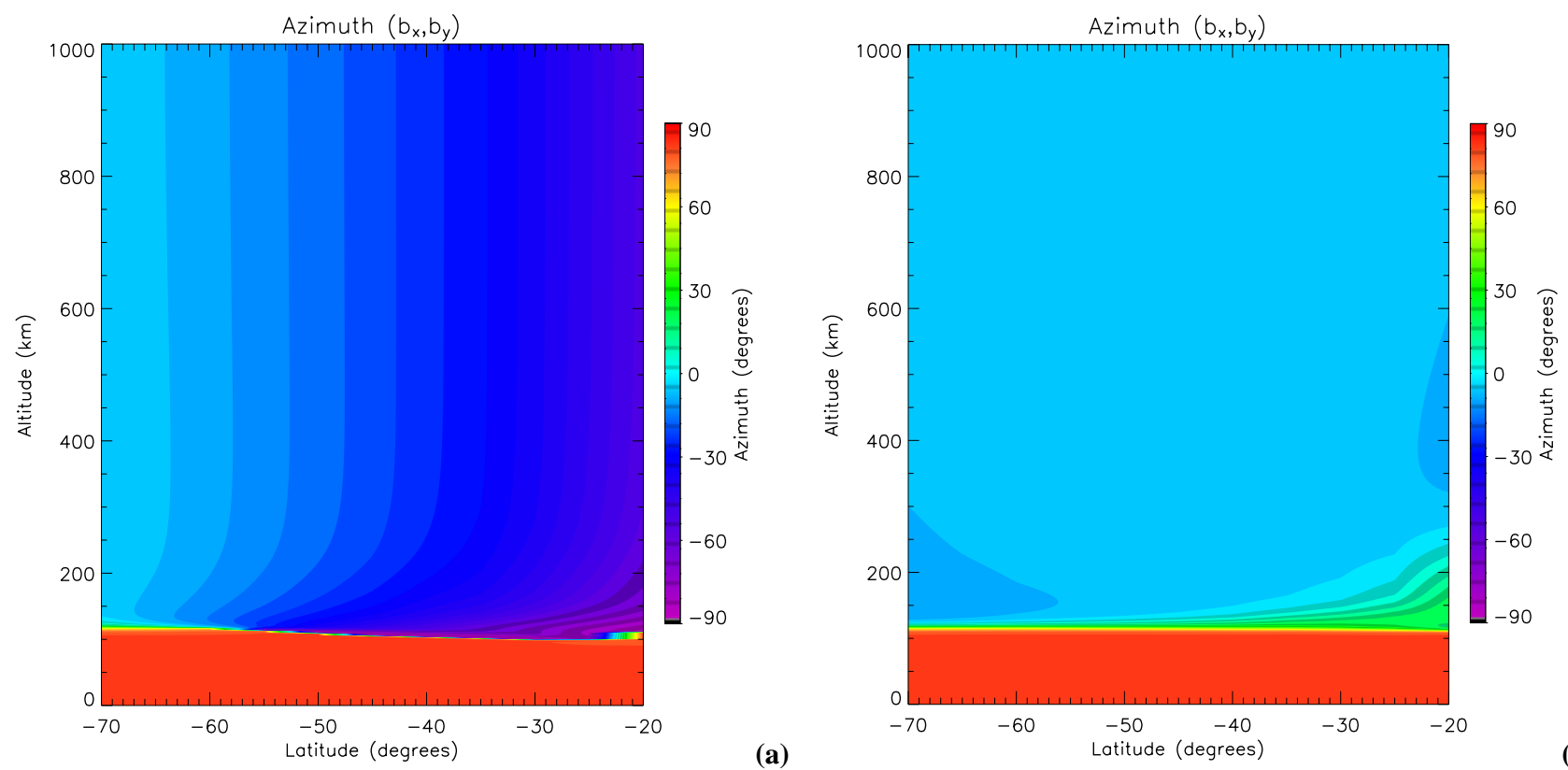

(b)

Fig. 6. ULF wave polarisation azimuth computed from the $b_{x}$ and $b_{y}$ components for a frequency of $25 \mathrm{mHz}$. The IRI-95 model parameters were chosen for conditions on 28 September 2000 for the $160^{\circ}$ geographic longitude at 10:00 LT. The geographic latitudes vary between $70^{\circ}$ and $20^{\circ}$ south. The north-south wavenumber is $k_{x}=10^{-10} \mathrm{~m}^{-1}$. The azimuthal wavenumber, (a) $m=3$, (b) $m=12$.

maximum conditions, the results for solar minimum conditions were also computed and show similar features. Essentially, the reduced conductivities for solar minumum and the larger Alfven speeds shift the features described above to smaller values of $k_{\perp}$. For example, the most gradual change in the polarisation azimuth with altitude in Fig. $4 \mathrm{a}$ is for $k_{y} \approx 1 \times 10^{-6} \mathrm{~m}^{-1}$. For solar minimum, the figure appears essentially the same except that the corresponding change in the polarisation azimuth with altitude occurs around $k_{y} \approx 1 \times 10^{-7}$.

\section{Discussion}

In order to further explore wave polaristaion azimuth rotation for oblique $\boldsymbol{B}_{\mathbf{0}}$, we can compare the results with the analytic model described in Sciffer et al. (2004). The numerical model described in the present paper derives the ULF wave fields in a different and therefore independent manner to the analytic formulation presented by Sciffer et al. (2004). The analytic model may be used to verify the results of the numerical model, provide insights into the reflection properties of the ionosphere and examine the ULF wave fields in more detail. The analytic model was formulated to allow the separation of incident and reflected components of the shear Alfvén and compressional mode waves. It uses height integrated conductivities, replacing the ionosphere by a current sheet, so we expect the details around the ionosphere Eregion to be different compared with the numerical calculations. However, due to the wavelengths involved the analytic and numerical model results should be similar away from the ionosphere E-region. One further difference between the analytic compared with the numerical formulation concerns the frequency. For Pc1 studies $(>0.2 \mathrm{~Hz})$, the slab construction of the analytic model does not describe features that occur due to the variation of the topside ionosphere with altitude. However, such details can be described using the numerical model presented in this paper.

Taking the oblique $\boldsymbol{B}_{\mathbf{0}}$ case presented in Fig. 5, the corresponding results from the analytic model are shown in Fig. 7. The parameters were chosen to closely represent those used in Fig. 5. The current sheet was placed at $d=120 \mathrm{~km}$, a height corresponding to the ionosphere E-region and the ULF wave frequency is $16 \mathrm{mHz}$. The height integrated conductivities are $\Sigma_{d}=10^{6} \mathrm{~S}, \Sigma_{P}=6.2 \mathrm{~S}$ and $\Sigma_{H}=7.6 \mathrm{~S}$, which correspond with solar maximum conditions for the data in Fig. 5. A change in polarisation azimuth from the magnetosphere to the atmosphere is seen for all wavenumbers in Fig. 7 as the analytic model specifies an atmosphere with zero conduction current. The variation of polarisation azimuth and the field aligned component of the ULF wave with wavenumber and altitude for the numerical model (Fig. 5a) and the analytic model (Fig. 7) are similar. This confirms that the analytic model is a good approximation to the numerical model for these frequencies.

Figure 7 a may be divided into three regions according to the magnitude of $k_{y}$. The first region is for $1 \times 10^{-8} \leq k_{y} \leq 2 \times 10^{-7} \mathrm{~m}^{-1}$ where the compressional mode is either propagating or has a very large $e$ folding distance. The compressional mode amplitude does not reduce with altitude so there is very little variation of the field aligned magnetic component of the ULF wave with altitude as seen in 

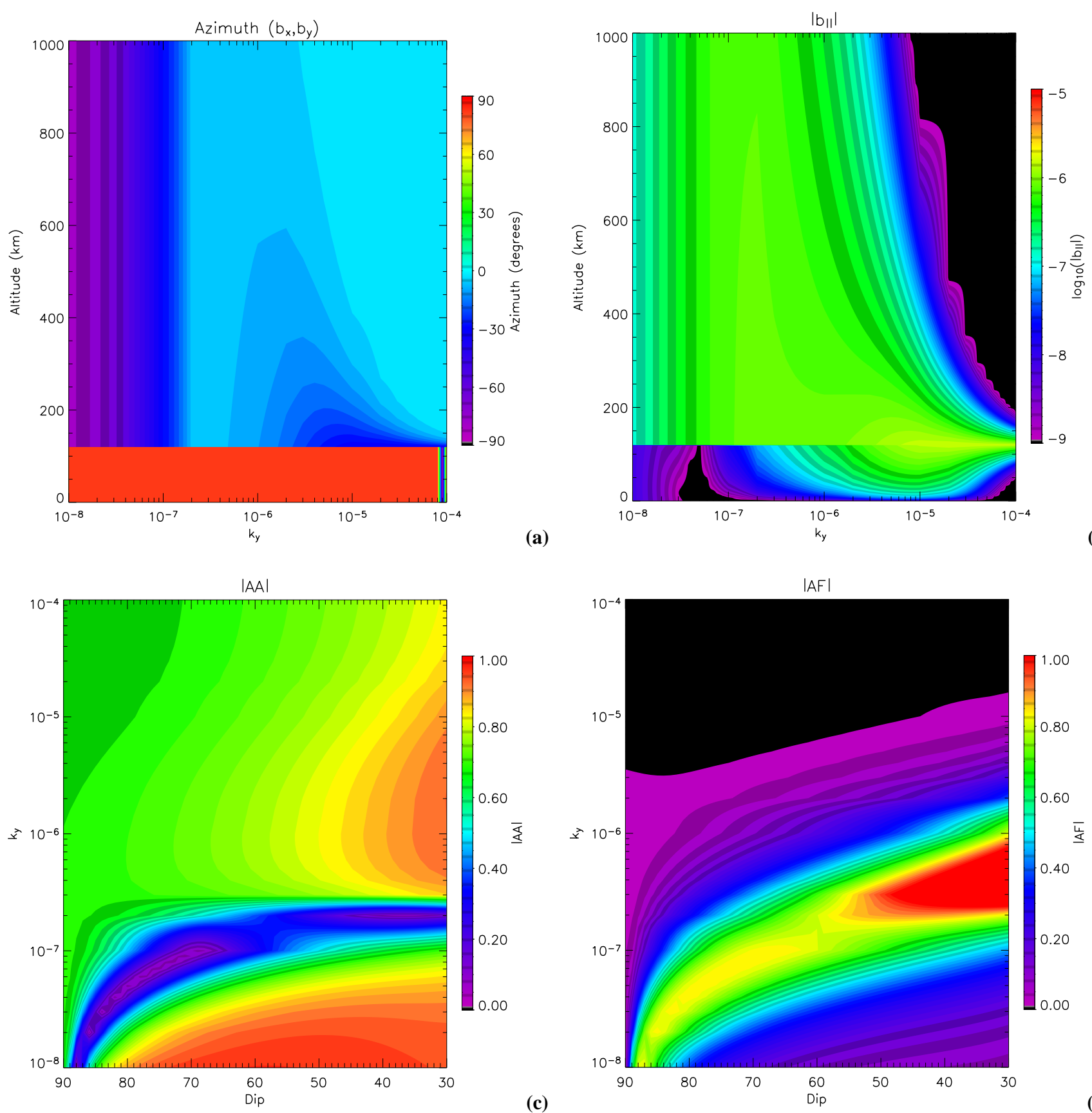

(a)

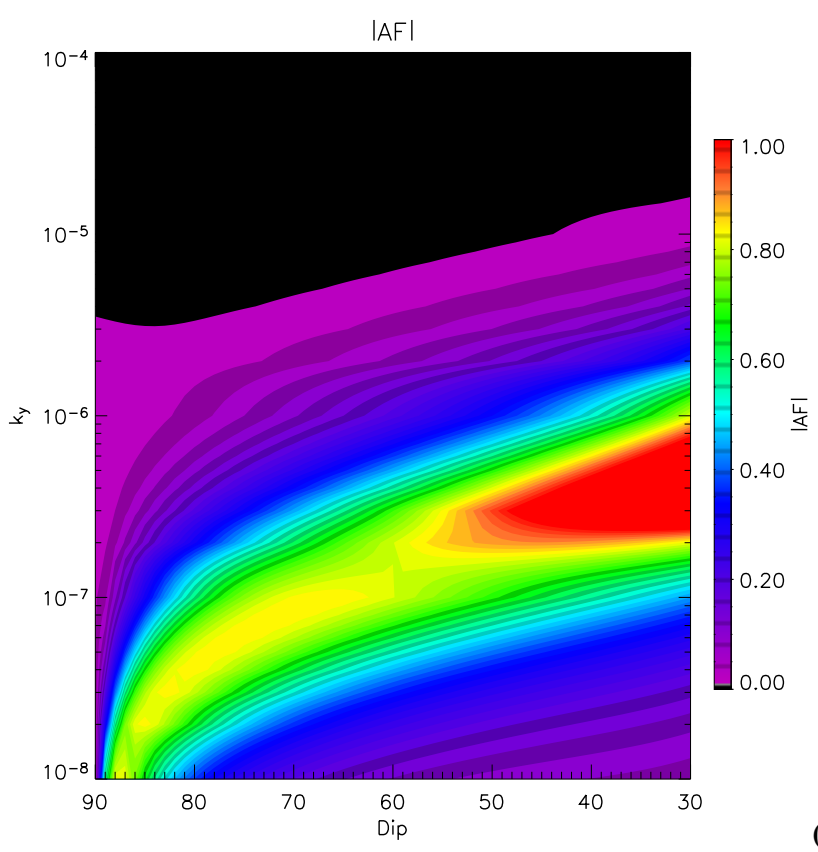

(b)

Fig. 7. Results from the analytical model of Sciffer et al. (2004). The parameters used were: $V_{a}=6.4 \times 10^{5} \mathrm{~ms}^{-1}, k_{x}=1 \times 10^{-10} \mathrm{~m}^{-1}$ and $k_{y}$ varying between $10^{-8} \mathrm{~m}^{-1}$ and $10^{-4} \mathrm{~m}^{-1}$. The current sheet altitude was $\mathrm{d}=120 \mathrm{~km}$ and the frequency, $16 \mathrm{mHz}$. The height integrated conductivities were $\Sigma_{0}=10^{6} \mathrm{~S}, \Sigma_{p}=6.2 \mathrm{~S}$ and $\Sigma_{h}=7.6 \mathrm{~S}$. (a) Polarisation azimuth rotation $\left(b_{x}, b_{y}\right)$ for dip, $I=70^{\circ}$. (b) Amplitude of the field aligned (compressional) component of the ULF wave for dip, $I=70^{\circ}$. (c) Absolute value of the reflection ratio, $A A$ versus $k_{y}$ and dip angle. (d) Same as (c) but for the conversion coefficient, $A F$ (shear Alfvén to compressional wave mode).

Fig. 7b. Therefore, there will be little change in azimuth with altitude as seen in Fig. 6a. Figures $7 \mathrm{c}$ and $7 \mathrm{~d}$ show the shear Alfvén wave reflection coefficient, $|A A|$ and the shear Alfvén to compressional mode conversion coefficient, $|A F|$ coefficients from Sciffer and Waters (2002) as a function of $k_{y}$ and dip angle. At $-20^{\circ}$ latitude, the dip is $\approx 45^{\circ}$. For $1 \times 10^{-8} \leq k_{y} \leq 2 \times 10^{-7} \mathrm{~m}^{-1}$, the conversion from shear
Alfvén to compresional mode, $|A F|$, varies between around 0.5 to 1 . This is the source of the non-NDR in azimuth seen at the lower latitudes in Fig. 6a. Over the range of $k_{y}$ that correspond with the latitudes in Fig. 6a, $|A F|$ increases for the lower latitudes (smaller wavenumbers). This places more compressional mode into $b_{y}$, reducing the magnitude of the azimuth rotation computed from $b_{x}$ and $b_{y}$ from the 
magnetosphere compared with the ground. In the case of a vertical $\boldsymbol{B}_{\mathbf{0}}$ the conversion coeffecient, $|A F|$ is very small and hence there is very little compressional component for small $k_{y}$ in Fig. $4 \mathrm{~b}$.

The second region in Fig. 7 is for $2 \times 10^{-7} \leq k_{y} \leq 2 \times 10^{-5} \mathrm{~m}^{-1}$. The effect on the ULF wave polarisation azimuth depends on the relative magnitudes of each ULF wave mode. For $k_{x} \approx 0$ the shear Alfvén mode appears mostly in $b_{x}$ while the compressional mode shows up in $b_{y}$ and $b_{z}$. Figure 8 shows in detail the ULF magnetic wave fields for $k_{y}=4 \times 10^{-6} \mathrm{~m}^{-1}$ where $|A A|=0.7$ and $|A F|=0.6$. For the polarisation azimuth computed from $b_{x}$ and $b_{y}$, we see that the shear Alfvén mode $\left(b_{x}\right)$ is constant with altitude above the ionosphere while the compressional mode $\left(b_{y}\right)$ decays exponentially upward. This is the reason for the more gradual change in polarisation azimuth with altitude seen in the second region of Figs. 7 and $3 \mathrm{~b}$. As $k_{y}$ increases the compressional mode decays more severely with altitude. In the third region of Fig. 7, where $k_{y}>2 \times 10^{-5}$, the compressional mode decreases in magnitude in a short distance from the ionosphere E-layer and no longer influences the polarisation azimuth.

So far we have only considered varying the wavenumber in the east-west direction, holding $k_{x} \approx 0$. Figure 9 shows the effect on the polarisation azimuth for different values of $k_{x}$ while $k_{y} \approx 0$, computed from the numerical model. Comparing this with Fig. 5, the azimuth rotation is similar to the $k_{y}$ dependent case. However, the amount of field aligned ULF wave magnetic perturbation, $\left|b_{\|}\right|$is quite different. For small wavenumbers, $\left|b_{\|}\right|$is significantly reduced above the ionosphere E-region, while in the atmosphere, $\left|b_{\|}\right|$is large. Using the analytic model, the conversion coefficient, $|A F|$ (shear Alfv'en to compressional mode) is 0.05 , which accounts for the small compressional mode amplitude above the ionosphere E-region. The reflection coeffecient for the shear Alfvén mode, $|A A|=0.85$. Therefore, the angle of arrival (changing the relative magnitudes of $k_{x}$ and $k_{y}$ ) has a significant effect on the amount of compressional mode that appears just above the ionosphere. In the atmosphere, the $b_{\|}$ signal comes from the magnetosphere $b_{x}$ contribution due to the oblique $\boldsymbol{B}_{\mathbf{0}}$. This may be understood from Fig. 8 where the fields are shown for the case where $k_{x} \approx 0$. For $k_{y} \approx 0$, the $b_{x}$ and $b_{z}$ components of the shear Alfvén mode reduce in magnitude to the $b_{y}$ level in Fig. 8 while the $b_{y}$ component increases to the magnitude of $b_{x}$ in Fig. 8. The shear Alfvén mode has most of its amplitude in $b_{y}$ for $k_{y} \approx 0$. Therefore, the compressional mode that appears has similar magnitude in $b_{z}$ but $b_{x}$ is large for $k_{y} \approx 0$ while $b_{y}$ is large for $k_{x} \approx 0$. The projection of the large $b_{x}$ onto the $\boldsymbol{B}_{\mathbf{0}}$ direction for $k_{y} \approx 0$ is the source of the larger field aligned component in the atmosphere.

Polarisation azimuth rotation for ULF waves that encounter the ionosphere has historically addressed the shear Alfvén mode with its associated field aligned current. Both wave modes are usually involved when computing the polarisation azimuth from $b_{x}$ and $b_{y}$ near the ionosphere. Does the compressional mode exhibit similar behavior? The structure
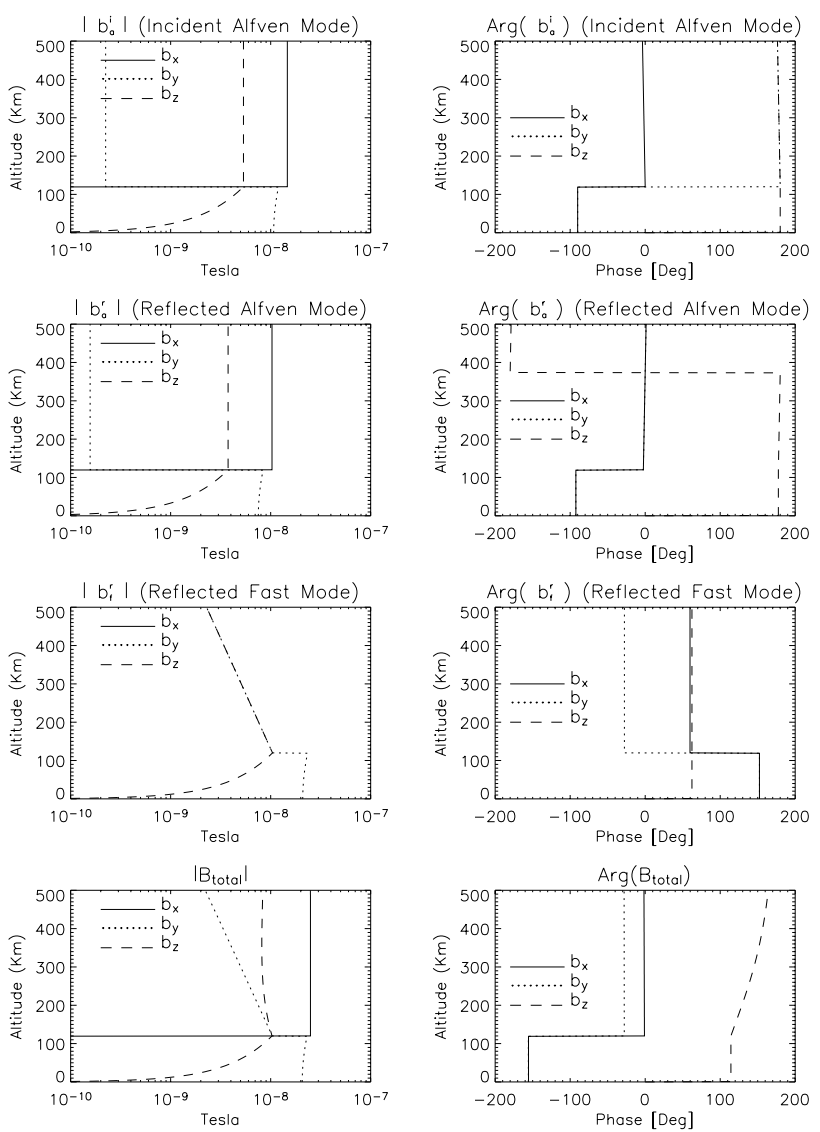

Fig. 8. Separation of the ULF magnetic wave fields using the analytical model of Sciffer et al. (2004). The parameters are the same as Fig. 7 but with $k_{y}=4 \times 10^{-6} \mathrm{~m}^{-1}$. Left-hand-panels show the magnitude of the respective wave fields while the right-handpanels show the phasing.

of the compressional mode for ideal MHD conditions is shown in Fig. 10 for $k_{y}=0$. The mode has perturbation current perpendicular to both $\boldsymbol{b}$ and $\boldsymbol{k}$, and parallel to $\boldsymbol{e}$. For $k_{y} \approx 0$, the shear Alfvén mode is confined to $b_{y}$ and the compressional mode to $b_{x}$ and $b_{z}$. Hence we can see that for a compressional mode in the magnetosphere with $k_{y}=0, \nabla \times \mathbf{b}$ is in the $y$ direction. In the neutral atmosphere, the wave magnetic field must either be zero or aligned along $\mathbf{k}$, which is in the $x z$ plane. If conversion to the shear Alfvén mode is zero, the rotation of the magnetic wave field is confined to the $x z$ plane. Therefore, there is no NDR for the compressional mode in the $x y$ and $y z$ planes. The compressional mode reflection coefficient is $\approx-1$ so the incident and reflected $b_{z}$ components cancel while the incident and reflected $b_{x}$ components add, giving no NDR in the $x z$ plane either. Our numerical and analytic model results confirm that there is no NDR in any plane for the case where we have a propagating compressional mode and no incident shear Alfvén mode. In these runs the conversion from compressional to shear Alfvén mode, $|F A|$ was around 0.01 giving a small $b_{y}$ component. Since $k_{x}<\frac{\omega}{V_{a}}$ for a propagating compressional mode, the reflected shear Alfvén mode does cause a small 

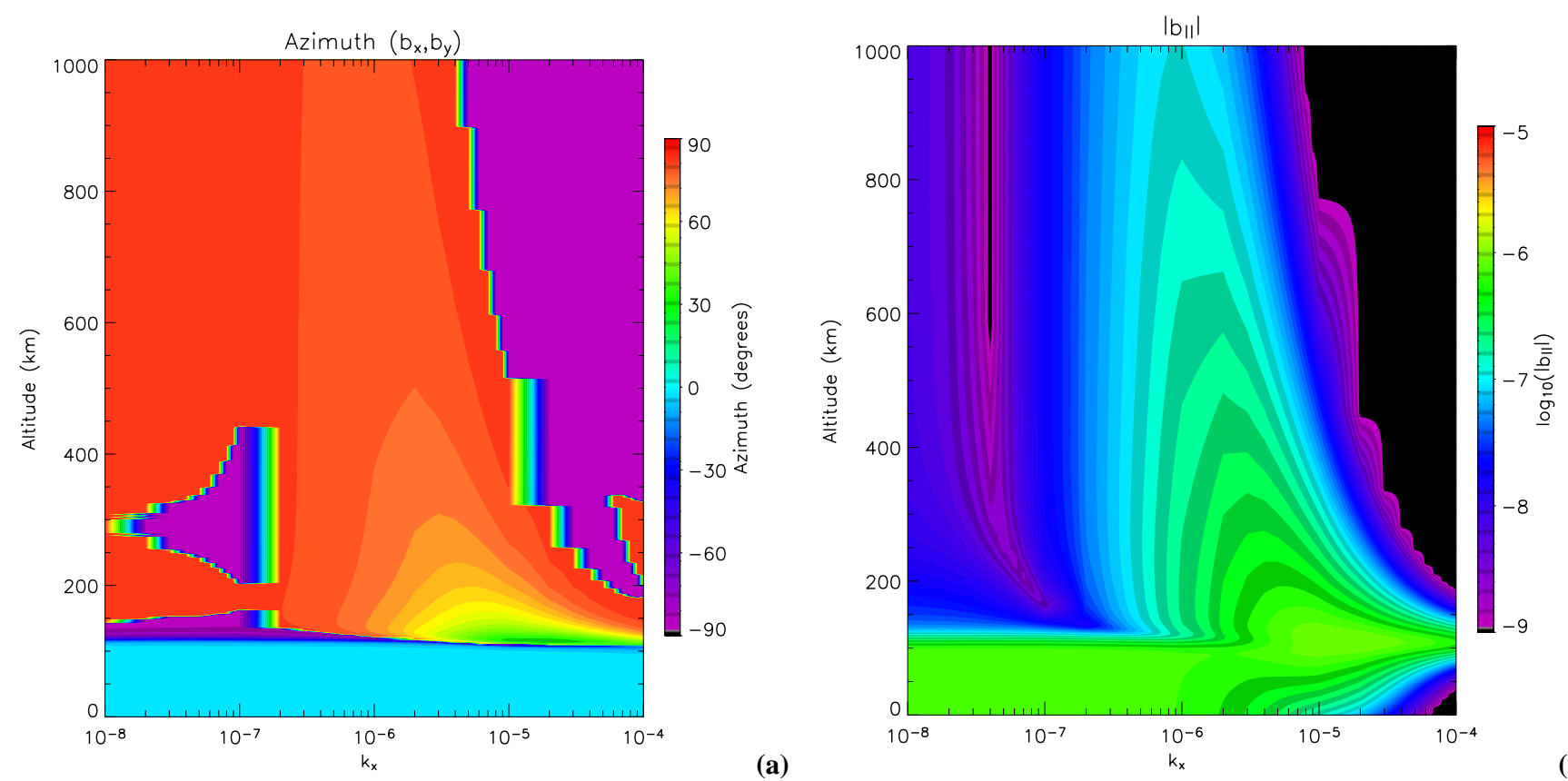

(b)

Fig. 9. The (a) polarisation azimuth computed from the $b_{x}$ and $b_{y}$ ULF wave fields and (b) the field aligned component of the ULF wave field for a frequency of $16 \mathrm{mHz}$, dip angle $I=70^{\circ}$ for solar maximum parameters. The east-west wavenumber, $k_{y}=1 \times 10^{-10} \mathrm{~m}^{-1}$.

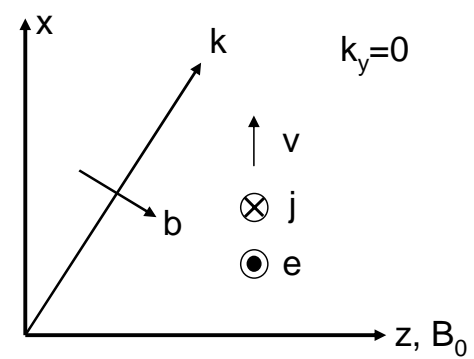

Fig. 10. The structure of a compressional ULF wave mode for ideal MHD and $k_{y}=0$ (Cross, 1988).

azimuth change in the $y z$ plane. For a mix of incident, propagating wave modes, $k_{z, f}$ is larger than $k_{x}$ to ensure the compressional mode propagates and a NDR appears in the $y z$ plane.

A polarisation azimuth rotation can appear for the incident compressional mode case if $\mathbf{k}$ varies in the $y$ direction which is perpendicular to the plane of $\mathbf{B}_{\mathbf{0}}$. A representative example is shown in Fig. 11 where the dip angle is $70^{\circ}, k_{x}=1 \times 10^{-10}$ for a wave frequency of $16 \mathrm{mHz}$. The polarisation azimuth in the atmosphere compared with that above the ionosphere Eregion $(120 \mathrm{~km})$ changes in the $x y$ and $x z$ planes for small $k_{y}$. For $k_{y}=4 \times 10^{-8} \mathrm{~m}^{-1}$ the incident compressional mode has field perturbations in all three components with $b_{x} \approx b_{y}$ and $b_{z} \approx 0.1 b_{x}$. The compressional mode reflection coefficient, $|F F| \approx 0.1$ while the compressional to shear Alfvén conversion, $|F A| \approx 0.8$. The resulting magnetic fields of the wave in the atmosphere are $b_{x} \approx b_{z}$ with $b_{y} \approx 100 b_{x}$. Therefore, the reduction in $b_{x}$ amplitude from the magnetosphere to the atmosphere results in polaristion azimuth rotation in the $x y$ and $x z$ planes as seen in Fig. 11 .

Compressional mode wave energy is generally thought to enter the magnetosphere from external sources such as the Kelvin-Helmholtz instability in the vicinity of the magnetopause (Southwood, 1968) or upstream ion-cyclotron wave generation (Yumoto, 1985). Propagation of compressional mode energy from the magnetosphere to the ionosphere and ground is hampered by wave propagation cutoffs in the magnetosphere (Zhu and Kivelson, 1989). This does not necessarily preclude the existence of propagating compressional mode waves around $1000 \mathrm{~km}$ altitude. Compressional mode energy may "tunnel" and appear as a propagating mode in the inner magnetosphere (Allan et al., 1986; Waters et al., 2000). Furthermore, an incident shear Alfvén wave that mode converts in the ionosphere may produce an upward propagating compressional mode as described in the context of Figs 4, 5, 7 , and 9. If the compressional mode does not decay sufficiently with increasing altitude and appears in the top side ionosphere, then an azimuth rotation in the vertical plane does occur but is not necessarily $90^{\circ}$.

\section{Conclusions}

The polarisation azimuth rotation of ULF waves in the horizontal plane $\left(b_{x}, b_{y}\right)$ from the magnetosphere through to the atmosphere does occur for oblique $\boldsymbol{B}_{\mathbf{0}}$. However, this varies with altitude. For compressional energy that is strongly attenuated with altitude, the NDR occurs over a 

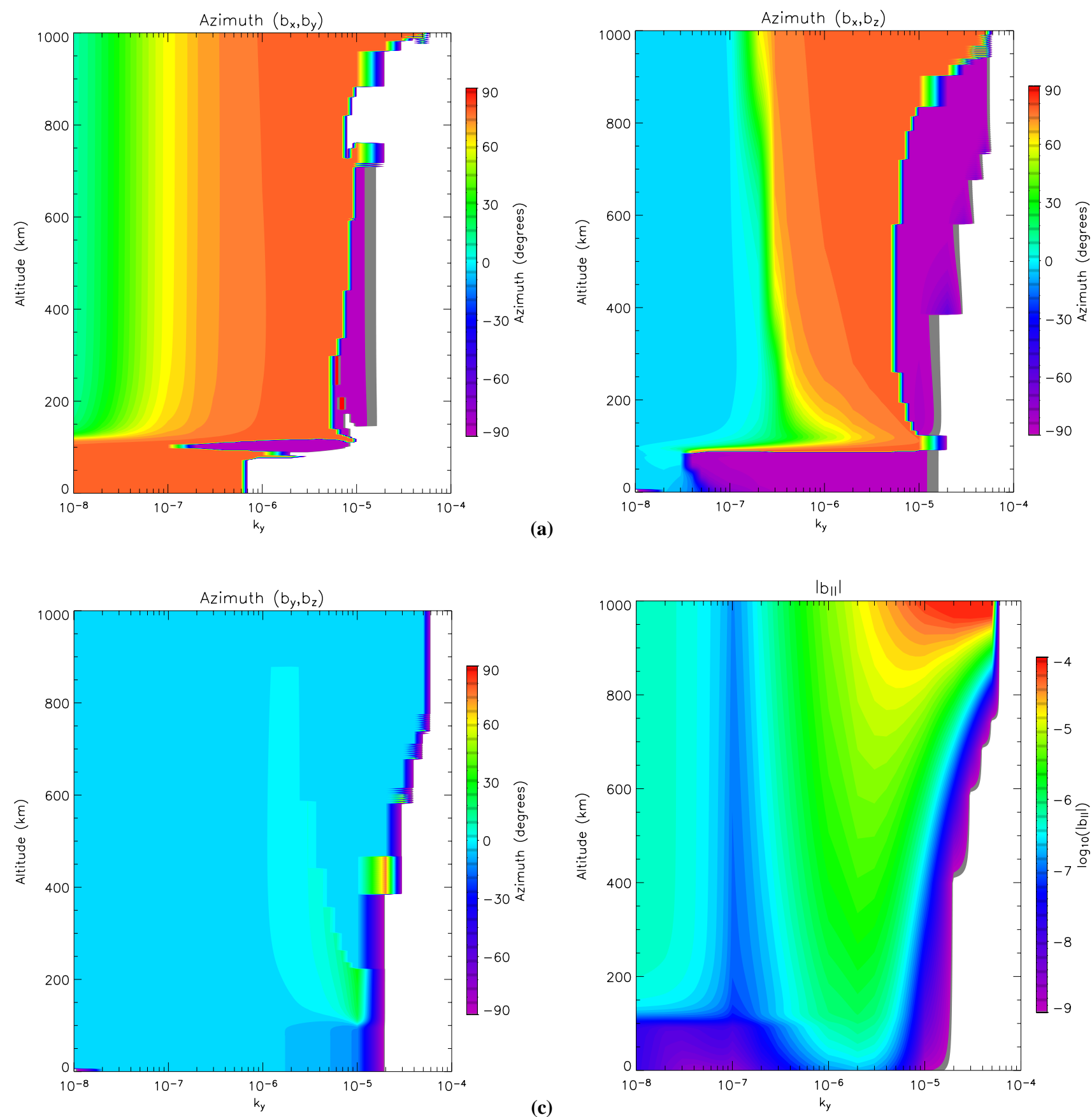

(b)

(c)

Fig. 11. Polarisation azimuths for an incident compresional (fast) mode. The ULF frequency was $16 \mathrm{mHz}$, the dip angle, $I=70^{\circ}$ and solar maximum conditions. Wavenumbers are $k_{x}=10^{-10} \mathrm{~m}^{-1}$ and $k_{y}$ varying between $10^{-8} \mathrm{~m}^{-1}$ and $10^{-4} \mathrm{~m}^{-1}$. (a) Polarisation azimuth for $b_{x}$ and $b_{y}$, (b) polarisation azimuth for $b_{x}$ and $b_{z}$ and (c) polarisation azimuth for $b_{y}$ and $b_{z}$, (d) the amplitude of the field aligned (compressional) component of the ULF wave magnetic field.

small altitude range, close to the ionospheric E-region. If the compressional energy propagates or has a large $e$ folding distance, then the polarisation azimuth in the top side ionosphere or magnetosphere compared with the atmosphere can be any value, as seen in the left portion of Fig. 7d. These conditions may be satisfied for low latitudes and small $\mathrm{m}$ numbers (Fig. 6a). Between these cases, the polarisation azimuth may take up to $500 \mathrm{~km}$ to rotate the full $90^{\circ}$. The altitude range is governed by the compressional mode characteristics and the mode conversion and reflection properties from the ionosphere. The current associated with the compressional mode wave also gives rise to a polarisation 
azimuth rotation effect but in the vertical plane, involving $b_{x}, b_{z}$ for $k_{y} \approx 0$. For $k_{x} \approx 0$, the compressional wave energy appears in all 3 spatial components. However, in all cases, as was pointed out by Southwood and Hughes (1983), the $\nabla \times \boldsymbol{b}$ changes from non-zero in the magnetosphere to zero in the atmosphere. This may or may not be manifested as a rotation of polarisation azimuth computed from the wave magnetic field components.

The numerical model described in this paper assumes a 1-D, horizontally uniform ionosphere, an approximation used in previous studies. We have focussed on the effects introduced by different magnetic field dip angles with latitude. The effect of the dip angle is introduced into the model via the conductivity tensor, $\bar{\sigma}$. Since ULF wave spatial scale sizes may be up to thousands of $\mathrm{km}$, the assumption of horizontal uniformity in $\bar{\sigma}$ over these distances is more reasonable for the azimuthal direction (provided the dawn and dusk longitudes are avoided) compared with the latitudinal direction. In order to fully quantify the validity of these assumptions, the results need to be compared with those from a higher dimensional model, a task presently underway.

\section{Appendix}

The direct, Pedersen and Hall conductivities of an ionised gas can be derived from equations of motion of the plasma. They may be expressed as,

$$
\begin{aligned}
\sigma_{0} & =\frac{1}{B_{0}} \sum_{i} \frac{n_{i} \Omega_{i}}{\bar{v}_{i}}\left|q_{i}\right| \\
\sigma_{1} & =\frac{1}{B_{0}} \sum_{i} \frac{n_{i} \Omega_{i}^{2} v_{i}}{\bar{v}_{i}^{2}+\Omega_{i}^{2}}\left|q_{i}\right| \\
\sigma_{2} & =-\frac{1}{B_{0}} \sum_{i} \frac{\Omega_{i}^{2} q_{i}}{\bar{v}_{i}^{2}+\Omega_{i}^{2}}\left|q_{i}\right|
\end{aligned}
$$

where $n_{i}, \Omega_{i}$, and $q_{i}$ are the number density, gyrofrequency and charge of the $i^{\text {th }}$ particle species. $\bar{v}_{i}=v_{i}-i \omega$ for the collision frequency, $v_{i}$, and the characteristic frequency, $\omega$ of the disturbance. $B_{0}$ is the magnitude of the background magnetic field. The collision frequencies may be calculated from the number densities of the given species together with the temperature, mean molecular mass and density of the neutral components (Kelley, 1989). The values required for these calculations were obtained from the IRI95 and MSISE90 models. The IGRF model was used for magnetic field values as well as dip angle for a particular locality. The values for the conductivities were calculated in $2 \mathrm{~km}$ increments from $80 \mathrm{~km}$ to $1000 \mathrm{~km}$.

The values below $80 \mathrm{~km}$ (or where the IRI and MSIS model had no data) were reduced with decreasing altitude, starting with the lowest altitude value. The direct and Pedersen conductivities were decreased using an $e$ folding distance of $6 \mathrm{~km}$ in the original model of Hughes (1974).
In general, the direct and Pedersen conductivities were chosen to decrease with an $e$ folding distance of $3 \mathrm{~km}$ which produced a smaller conductivity near the ground $\left(\approx 2 \times 10^{-14} \mathrm{~S} / \mathrm{m}\right)$. The Hall conductivity has an $e$ folding distance set to $1 \mathrm{~km}$ (e.g. Zhang and Cole, 1994).

Acknowledgements. This work was supported by grants from the Australian Research Council, the University of Newcastle and the Cooperative Research Centre for Satellite Systems. The authors wish to thank P. Ponomarenko for helpful discussions.

Topical Editor M. Pinnock thanks W. J. Hughes and another referee for their help in evaluating this paper.

\section{References}

Allan, W., Poulter, E., and White, S.: Hydromagnetic wave coupling in the magnetosphere- Plasmapause effects on impulseexcited resonances, Planet. Space Sci., 34, 1189-1200, 1986.

Cross, R.: An introduction to Alfvén waves, Addison Wesley, Massachusetts, 1988.

Hedin, A. E.: A revised thermospheric model based on mass spectrometer and incoherent scatter data: MSIS-83, J. Geophys. Res., 88, $10170-10188,1991$.

Hughes, W. J.: The effect of the atmosphere and ionosphere on long period magnetospheric micropulsations, Planet. Space Sci., 22, 1157-1172, 1974.

Hughes, W. J. and Southwood, D.: The screening of micropulsation signals by the atmosphere and ionosphere, J. Geophys. Res., 81, 3234-3240, 1976.

Kelley, M. C.: The Earth's Ionosphere - Plasma Physics and Electrodynamics., Academic Press Inc., 1989.

Olson, J. V. and Rostoker, G.: Longitudional phase variation of Pc 4-5 micropulsations., J. Geophys. Res., 83, 2481-2488, 1978.

Pitteway, M. L. V.: The numerical calculation of wave fields, reflection coefficients and polarizations for long radio waves in the lower ionosphere., Roy, Soc. Phil. Trans., 257, 219-239, 1965.

Poole, A. W. V., Sutcliffe, P. R., and Walker, A. D. M.: The relationship between ULF geomagnetic pulsations and ionospheric doppler oscillations: Derivation of a model., J. Geophys. Res., 93, 14656-14664, 1988.

Sciffer, M. D. and Waters, C. L.: Propagation of ULF waves through the ionosphere: Analytic solutions for oblique magnetic fields, J. Geophys. Res., 107, 1297-1311, 2002.

Sciffer, M. D., Waters, C. L., and Menk, F. W.: Propagation of ULF waves through the ionosphere: Inductive effect for oblique magnetic fields, Ann. Geophys., 22, 1155-1169, 2004, SRef-ID: 1432-0576/ag/2004-22-1155.

Southwood, D. J.: The hydromagnetic stability of the magnetospheric boundary, Planet. Space Sci., 16, 587-605, 1968.

Southwood, D. J. and Hughes, W. J.: Theory of hydromagnetic waves in the magnetosphere, Space Sci. Rev., 35, 301-366, 1983.

Stix, T. H.: The theory of plasma waves., McGraw-Hill, New York, 1962.

Waters, C. L., Harrold, B. G., Menk, F. W., Samson, J. C., and Fraser, B. J.: Field line resonances and waveguide modes at low latitudes: 2. A model, J. Geophys. Res., 105, 7763-7774, 2000.

Yoshikawa, A. and Itonaga, M.: The nature of reflection and mode conversion of MHD waves in the inductive ionosphere: Multistep mode conversion between divergent and rotational electric fields, Geophys Res. Lett., 105, 10 565-10 584, 2000. 
Yoshikawa, A., Obana, Y., Shinohara, M., Itonaga, M., and Yumoto, K.: Hall-induced inductive shielding effect on geomagnetic pulsations, Geophys. Res. Lett., 9, 8, doi:10.1029/2001GL013610, 2002.

Yumoto, K.: Low-Frequency upstream wave as a probable source of low-latitude Pc3-4 magnetic pulsations, Planet. Space Sci., 33, 239-249, 1985.

Zhang, D. Y. and Cole, K. D.: Some aspects of ULF electromagnetic wave relations in a stratified ionosphere by the method of boundary value problem., J. Atmos. Terr. Phys., 56, 681-690, 1994.
Zhang, D. Y. and Cole, K. D.: Formulation and computation of hydromagnetic wave penetration into the equatorial ionosphere and atmosphere, J. Atmos. Terr. Phys., 57, 813-819, 1995.

Zhu, X. and Kivelson, M. G.: Global mode ULF pulsation in a magnetosphere with a nonmonotonic Alfv́en velocity profile, J. Geophys. Res., 94, 1479-1485, 1989. 